\title{
Analysis of Energetic Particle Confinement in LHD Using Neutron Measurement and Simulation Codes ${ }^{*}$
}

\author{
Hideo NUGA $^{1)}$, Ryosuke SEKI ${ }^{1,2)}$, Kunihiro OGAWA ${ }^{1,2)}$, Shuji KAMIO ${ }^{1)}$, Yutaka FUJIWARA ${ }^{1)}$, \\ Masaki OSAKABE ${ }^{1,2)}$, Mitsutaka ISOBE ${ }^{1,2)}$, Takeo NISHITANI ${ }^{1)}$, Masayuki YOKOYAMA ${ }^{1,2)}$ \\ and LHD Experiment Group \\ 1) National Institute for Fusion Science, National Institutes of Natural Sciences, Toki 509-5292, Japan \\ 2) SOKENDAI (The Graduate University for Advanced Studies), Toki 509-5292, Japan
}

(Received 10 January 2019 / Accepted 7 March 2019)

\begin{abstract}
The fast ion confinement time in the Large Helical Device (LHD) is investigated by using the neutron measurement and simulations. To estimate the fast ion confinement time, a series of short pulse neutral beam (NB) injection experiments have been performed. Additionally, the NB heating simulation code, CONV_FIT3D, has been extended to estimate the neutron emission rate in LHD. We estimate the fast ion confinement time from the differences between the measured and the simulated neutron decay times after the NB is turned off. It is found that the fast ion confinement time of three tangential NBs have similar values $\tau_{c} \sim 0.5 \mathrm{sec}$ and the confinement time of the perpendicular NB is approximately $\tau_{c} \sim 0.06 \mathrm{sec}$. Additionally, by using the confinement time and the ion ratio data estimated from the simulation result, we can obtain a simulation result similar to the measured data.

(c) 2019 The Japan Society of Plasma Science and Nuclear Fusion Research
\end{abstract}

Keywords: fast ion confinement, neutron emission rate, LHD

DOI: $10.1585 /$ pfr. 14.3402075

\section{Introduction}

In magnetic confinement fusion devices, the plasma heating is achieved by the kinetic energy transfer from the fast ions, which is generated by the neutral beam injection, radio frequency waves, and fusion reactions, to the bulk plasmas. Therefore, the high performance of the fast ion confinement leads to the high efficiency of the plasma heating. For this reason, the confinement of the fast ions has been investigated in several magnetic confinement fusion devices and in simulations.

The deuterium plasma experiment in the Large Helical Device (LHD) started in March 2017 [1-4]. In general, deuterium fusion plasmas yield fast neutrons due to the deuterium-deuterium (DD) fusion reaction. Because the neutron measurement plays an important role in the studies of fast ion confinement, LHD is equipped with the neutron diagnostics systems [5]. In the present fusion devices, such as LHD, the fusion reaction between thermal ion and accelerated fast ion is dominant instead of the reaction between thermal ions. The DD fusion cross-section monotonically increases with the increase of the fast ion velocity within the range of the typical neutral beam (NB) energy $(\lesssim 200)$ in LHD. Therefore, the neutron emission rate reflects the fast ion density profile and the fast ion velocity distribution. In other words, we can investigate the

author's e-mail: nuga.hideo@nifs.ac.jp

*) This article is based on the presentation at the 27th International Toki Conference (ITC27) \& the 13th Asia Pacific Plasma Theory Conference (APPTC2018). fast ion transport and the slowing down from the neutron measurement.

In this paper, we investigate the fast ion confinement time in LHD by using the neutron measurement. Generally, the fast ion transport mechanism is very complex. This is because the transport mechanisms consist of several physics, such as the classical Coulomb collisional diffusion, the neo-classical transport due to inhomogeneous magnetic field, and the wave-induced transport. The accurate simulation including these phenomena requires immense computational resources. In this research, instead of an accurate analysis, we estimate the fast ion confinement time from the analysis of the experiment data. As noted above, the neutron emission rate has information about the fast ion transport and the slowing down. Although the estimation of the fast ion transport is difficult due to its complexity, the fast ion slowing down process can be estimated by using several simulation codes. Therefore, we estimate the fast ion confinement time from the differences between the neutron measurement and the simulation result, which ignores the fast ion transport during fast ion slowing down process.

The rest of this paper consists of the following sections. The introduction of the experiment scenario and the setup are provided in section 2 . Analysis tools and simulation models used in this paper are explained in section 3. A benchmark test is also provided in section 3. Experiment and analysis results are shown in section 4 . The conclusion 
of this paper appears in section 5 .

\section{Experimental Setup}

\subsection{Scenario}

To estimate the fast ion confinement time, a series of short pulse NB injection experiments have been performed. We have observed the fusion-born fast neutrons and the decay time of the neutron emission rate after the NBIs have been turned off. Because short pulse NBs have insignificant impact on the bulk plasma, fluctuations of the plasma parameters can be suppressed and fast deuterons will slow down under constant conditions. Figure 1 shows the typical waveforms of this experiment. Neutral beam port-through power and injection energy in each beam, electron temperature and density on axis, and the neutron emission rate are displayed. NB\#1 to NB\#4 are injected with the pulse width of $40 \mathrm{~ms}$. In addition, plasmas are heated by $2.5 \mathrm{MW}$ electron cyclotron heating system from $3.25 \mathrm{sec}$ to $5.25 \mathrm{sec}$ in order to sustain the plasmas.

It can be considered that the decay of the neutron emission rate after the NBIs are turned off comes from two factors. One is the reduction of the fusion cross-section due to the slowing down of the fast deuteron, and the other is the reduction of the fast deuteron density due to the fast deuteron transport. Therefore, the neutron decay time $\tau_{n}$ can be expressed as below:

$$
\tau_{n}^{-1}=\tau_{s}^{-1}+\tau_{c}^{-1}
$$

where $\tau_{s}$ is the $e$-folding time of the neutron emission rate owing to the fast deuteron slowing down and $\tau_{c}$ is the fast ion confinement time. Conventionally, the classical neutron decay time $\tau_{n}^{\mathrm{cl}}$ is often used $[6,7]$ for $\tau_{s} . \tau_{n}^{\mathrm{cl}}$ is expressed as:

$$
\begin{aligned}
\tau_{n}^{\mathrm{cl}} & =\frac{\tau_{s e}}{3} \ln \left(\frac{E_{0}^{3 / 2}+E_{C}^{3 / 2}}{E_{1}^{3 / 2}+E_{C}^{3 / 2}}\right), \\
\tau_{s e} & =\frac{3(2 \pi)^{3 / 2} \epsilon_{0}^{2} m_{D} T_{e}^{3 / 2}}{n_{e} e^{4} m_{e}^{1 / 2} \ln \Lambda},
\end{aligned}
$$

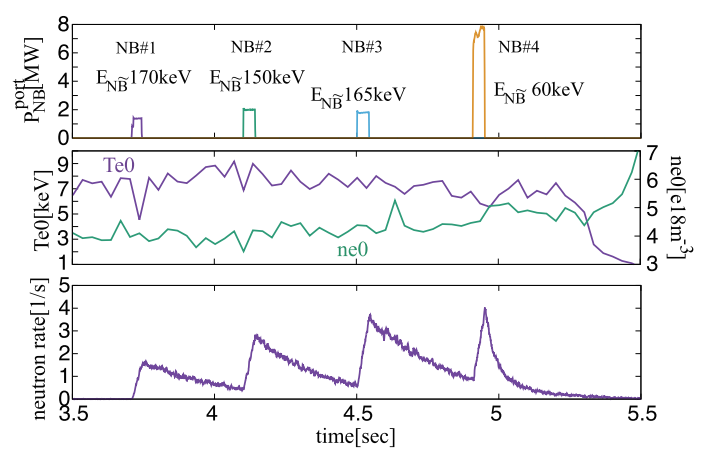

Fig. 1 Typical waveform of this series of experiments (SN139591). Neutral beam port-through power in each beam, electron temperature and density on axis, and the neutron emission rate are displayed. where $E_{0}$ is the injection energy, $E_{C}$ is the critical energy where the ion drag force is equal to the electron drag force, $m_{e}$ and $m_{D}$ are the rest mass of electron and deuteron, and $\tau_{s e}$ is the Spitzer slowing down time on electron. $E_{1}$ is the kinetic energy satisfying the following relation:

$$
\frac{\sigma\left(E_{1}\right) \sqrt{E_{1}}}{\sigma\left(E_{0}\right) \sqrt{E_{0}}}=\frac{1}{e},
$$

where $\sigma$ indicates the fusion cross section. In the following analysis, the neutron decay time simulated by CONV_FIT3D, which is introduced in section 3, is used for $\tau_{s}$. The simulated neutron decay time can take the plasma profiles into account.

In the present paper, we focus on the neutron decay time instead of the neutron emission rate itself. This is because the absolute value of the neutron emission rate is sensitive to uncertain parameters. In general, fusion reaction rate $\mathrm{R}$ can be described as:

$$
\mathcal{R}=\iint \sigma\left(E_{a b}\right) \bar{v} f_{a}\left(\boldsymbol{v}_{a}\right) f_{b}\left(\boldsymbol{v}_{b}\right) d \boldsymbol{v}_{a} d \boldsymbol{v}_{b}
$$

where $E_{a b}=0.5 m_{a} m_{b} /\left(m_{a}+m_{b}\right) \bar{v}^{2}$ is the relative kinetic energy, $\bar{v}=\left|\boldsymbol{v}_{a}-\boldsymbol{v}_{b}\right|$ is the relative velocity, and subscripts $a$ and $b$ indicate the particle species, respectively. When the beam-thermal fusion reaction is dominant, the expression of the fusion reaction rate can be reduced as

$$
\mathcal{R}_{b t}=n_{D} \int\langle\sigma v\rangle_{b t}\left(T_{i}, E_{a}\right) f_{a}\left(\boldsymbol{v}_{a}\right) d \boldsymbol{v}_{a}
$$

where $n_{D}$ indicates the bulk deuteron density, $T_{i}$ is the ion temperature, and $E_{a}$ is the kinetic energy of species " $a$." Although the fusion reaction rate is proportional to the bulk deuteron density, it is difficult to estimate the bulk deuteron density profile accurately due to the lack of impurity measurements. Therefore, the estimation of the absolute value of the neutron emission rate has an ambiguity. On the other hand, the neutron decay time has fewer ambiguities. Since fast deuterons slow down due to the fast deuteron-electron Coulomb collision, the slowing down of fast deuterons is not sensitive to the plasma effective charge [8]. Because of these reasons, we planned to perform the short pulse NB injection experiment and focused on the neutron decay time.

\subsection{Apparatus}

Figure 2 shows the top view of the NBI system in LHD. LHD is equipped with three tangential NBIs (NB\#1 -

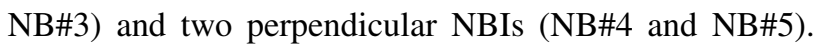
Beam ion species is deuteron. Typical value of beam injection energy and the port through power of NB\#1 - NB\#4 are illustrated in Fig. 2. In this paper, NB\#5 is not used. In the following experiments, the toroidal magnetic field strength is $|B|=2.75 \mathrm{~T}$, whose direction is counter-clockwise from the top view and the preset of the magnetic axis position is $R_{\text {axis }}=3.6 \mathrm{~m}$. Therefore, $\mathrm{NB} \# 1$ and $\mathrm{NB} \# 3$ are co-direction of the magnetic field and NB\#2 is counter-direction. The 


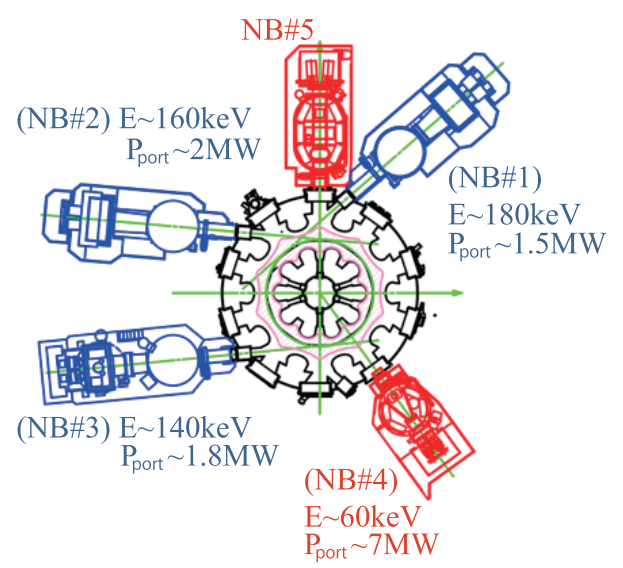

Fig. 2 Top view of NBI system in LHD is shown. NB\#1 to $\mathrm{NB} \# 3$ are tangential NBIs and $\mathrm{NB} \# 4$ and $\mathrm{NB} \# 5$ are perpendicular NBIs. In the present paper, NB\#5 is not injected.

averaged plasma minor radius is approximately $0.6 \mathrm{~m}$. The plasma temperature and density profiles have been measured by the Thomson scattering diagnostics and the neutron emission rate has been measured by Neutron Flux Monitor [9].

\section{Analysis Tools}

In this paper, simulation analyses of the neutron emission rate consist of two steps. The first step is the fast ion birth process and the second step is the fast ion slowing down process, respectively. The first step is simulated by FIT3D code [10-12]. FIT3D calculates the ionization of NB particles and the radial redistribution of fast ions due to the prompt orbit loss effect. This code takes the threedimensional magnetic equilibrium, the plasma density and temperature, the beam injection energy, and the beam portthrough power as input. The second step is simulated by CONV_FIT3D code. CONV_FIT3D code calculates the slowing down of NB fast ions in each normalized minor radius $\rho$. This code takes the fast ion birth profile, which is calculated by FIT3D, and plasma temperature and density as input. By using CONV_FIT3D, the energy distribution of NB fast deuterons can be obtained. Originally, this code has been developed to obtain NB heating profile. In the present paper, we have extended this code to calculate the neutron emission rate. The details of this code are provided in section 3.1 .

These two codes are components of the integrated transport analysis suite, TASK3D-a [13].

\subsection{CONV_FIT3D}

CONV_FIT3D calculates the time evolution of the kinetic energy of the test ions, which are injected every $\Delta t \sim 2 \mathrm{~ms}$. The test ion has information regarding the number of NB ions absorbed during $\Delta t$. This information, which is obtained as the simulation result of FIT3D, is taken as input. By accumulating the test ions until an arbitrary time $t$, the energy distribution at the time can be obtained roughly. The kinetic energy of test ions obeys the fast ion slowing down equation based on the classical Coulomb collision theory [14]:

$$
\begin{aligned}
& \frac{d E}{d t}=\frac{E}{\tau_{s e} / 2}\left(1+\frac{E_{c}^{1.5}}{E^{1.5}}\right), \\
& E_{c}=\left(\frac{3 \sqrt{\pi}}{4}\right)^{2 / 3} \frac{m_{b} T_{e}}{m_{e}^{1 / 3}}\left(\frac{Z}{m}\right)_{\mathrm{eff}}^{2 / 3}, \\
& \left(\frac{Z}{m}\right)_{\mathrm{eff}}=\sum_{j} \frac{n_{j} Z_{j}^{2}}{n_{e} m_{j}},
\end{aligned}
$$

where $E$ is the kinetic energy of test ions, $E_{c}$ is the critical kinetic energy where ion drag force is equal to the electron drag force, and the subscript " $j$ " indicates ion species, respectively.

In this paper, CONV_FIT3D is extended to calculate the neutron emission rate in LHD experiments. Within the typical plasma parameters on LHD, the fusion reaction between NB fast deuteron and thermal deuteron is dominant. Therefore we adopt the beam-thermal fusion cross-section $\langle\sigma v\rangle_{b t}$ derived by Mikkelsen [15]. The first term of eq. (7) in Ref. [15] is implemented in CONV_FIT3D. Since the typical plasma temperature in LHD is less than $10 \mathrm{keV}$, the reduced implementation is sufficiently accurate. The fitting coefficients for the astrophysical $S$ function used in $\langle\sigma v\rangle_{b t}$ are chosen from Ref. [16].

There are two notifications regarding the simulation model. At first, since CONV_FIT3D is a code to calculate the kinetic energy dissipation due to the fast ion slowing down, the pitch angle scattering and distribution are ignored. In general, this lack of information causes under-estimation of the neutron emission rate. This is because this model can not consider the fusion reaction between non-thermal particles. For example, if there are two tangential NBs which have an opposite direction to each other, the fusion reaction between two NB fast ions can not be ignored. However, in the present experiment, the fusion reaction between non-thermal ions can be neglected. This is because NBs are injected one by one, as shown in Fig. 1. The second notification is that the Coulomb collision model used in eq. (7) assumes that the velocity of test ions is sufficiently faster than that of thermal ion velocity. This assumption is broken in low energy region. Since the fusion cross-section in such a low energy region can be negligible, however, the Coulomb collision model is valid for the neutron estimation.

\subsection{Benchmark of the neutron estimation}

A benchmark test among a measurement in LHD and three simulation codes has been performed to validate the neutron emission rate estimation implemented in CONV_FIT3D. Here, FBURN [17] and TASK/FP [8] are chosen for benchmark codes. FBURN code has been developed for the time dependent analysis of DD and DT 
neutron emission rate. The Coulomb collision term implemented in FBURN is based on the classical fast ion slowing down theory, which is similar to CONV_FIT3D. TASK/FP is a three-dimensional (two dimensional in momentum space and one dimensional in radial direction) Fokker-Planck code. TASK/FP implements relativistic non-linear Coulomb collision model [18], which is the more rigorous model than the model implemented in CONV_FIT3D and FBURN. This Coulomb collision model can include the Coulomb collision effect between non-thermal ions and this model does not assume that the fast ion velocity is sufficiently faster than thermal ion velocity. In addition, TASK/FP can estimate the fusion reaction between non-thermal ions because TASK/FP can consider the pitch angle distribution.

Figure 3-(a) shows the waveform of NB port through power and Fig. 3-(b) shows the evolutions of measured and simulated neutron emission rates. In Fig. 3-(b), blue solid, green dotted, red dashed, and yellow dash-dotted curves denote the simulation results of FBURN, CONV_FIT3D, and two kinds of TASK/FP, respectively. The result of "TASK/FP full" uses eq. (5) for the fusion reaction rate. On the contrary, the result of "TASK/FP bt" uses eq. (6) for the fusion reaction rate. The difference between these two results comes from two contributions. One is the fusion reaction between thermal ions and the other is the fusion reaction between non-thermal ions. In this case, the fusion reaction rate between thermal ions is negligibly small. Therefore, the difference between "TASK/FP full" and "TASK/FP bt" indicates the contribution from the fusion reaction between non-thermal ions. This non-thermal contribution can not be estimated by CONV_FIT3D and FBURN. In this benchmark, it is assumed that the effective charge of the plasma is $Z_{\mathrm{eff}}=1$, the plasma is a fully ion-

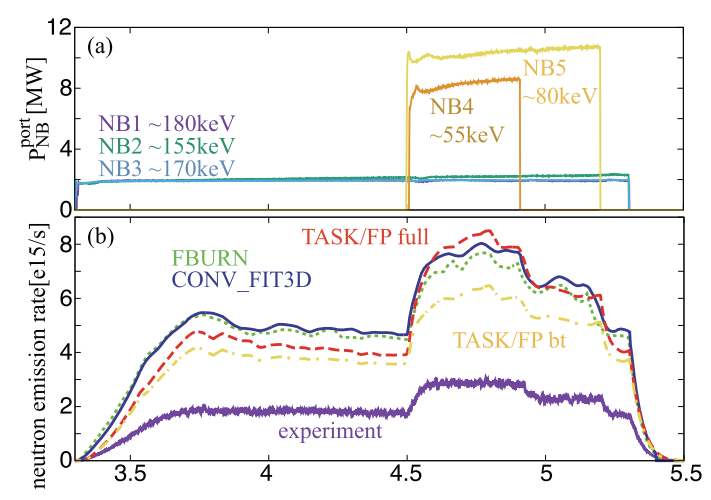

Fig. 3 The waveform of NB port through power and evolutions of the measured and simulated neutron emission rates are shown (SN140924). In figure (b), blue solid, green dotted, red dashed, and yellow dash-dotted curves denote the simulation results of FBURN, CONV_FIT3D, two kinds of TASK/FP, respectively. "TASK/FP full" includes beam-thermal and beam-beam fusion components and "TASK/FP bt" includes only beam-thermal component. ized pure deuteron plasma, and the ion temperature is equal to the electron temperature $T_{i}=T_{e}$. The absolute values of simulated neutron emission rate is over-estimated because of these assumptions and the lack of the fast ion transport.

From Fig. 3-(b) it is found that the results of FBURN and CONV_FIT3D have a good agreement. On the other hand, the results of TASK/FP are slightly smaller than that of CONV_FIT3D. In this benchmark, CONV_FIT, FBURN and "TASK/FP bt" use the same model [15] of the beam-thermal fusion cross section. Therefore, the differences of the neutron emission rate come from the differences of the fast deuteron energy distribution. It can be considered that the difference of the Coulomb collision model causes the the difference of the neutron estimation.

\section{Analysis Results}

Figure 4, which is the example of the analysis result, shows the NB port-through power and the time evolution of the measured (solid curve) and simulated (dotted curve) neutron emission rate. As noted in eq. (1), the simulated neutron decay time is longer than the measured neutron decay time due to the lack of the fast ion transport. Since the difference of these two neutron decay times indicate the fast ion confinement time, we investigated the relation of these two neutron decay times over 18 shots having different temperature and density. In these shots, the plasma temperature on $\rho=0.5$ has a range of $2<T_{e}[\mathrm{keV}]<$ 4.5 and the electron density on $\rho=0.5$ has a range of $0.5 \times 10^{19}<n_{e}\left[\mathrm{~m}^{-3}\right]<2.5 \times 10^{19}$. Figure 5 shows the relation between measured and simulated neutron decay times. The horizontal and vertical axes denote the simulated and measured neutron decay times, $\tau_{n}^{\text {sim }}$ and $\tau_{n}^{\text {exp }}$. These neutron decay times, $\tau_{n}^{\text {exp }}$ and $\tau_{n}^{\text {sim }}$, are estimated by the weighted least-square fitting in $S_{n}^{\text {peak }} \exp \left(-t / \tau_{n}\right)$, which is weighted by the variance of the measured neutron emission rate [5], where $S_{n}^{\text {peak }}$ is the peak value of the neutron emission rate. The y error-bars displayed in Fig. 5 indicate the standard deviation of $\tau_{n}^{\text {exp }}$. As the simulated neutron decay time increases, the simulated neutron decay times separate from the measured values. This is because in high $\tau_{n}^{\mathrm{sim}}$ region the contribution of the fast deuteron transport

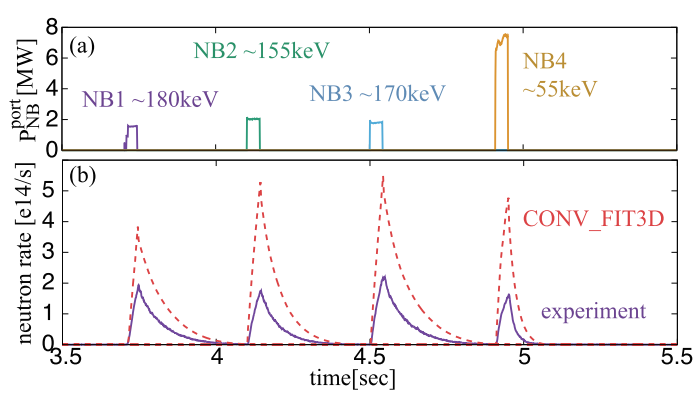

Fig. 4 The waveform of NB port through power and evolutions of the measured and simulated neutron emission rates are shown (SN139601). 


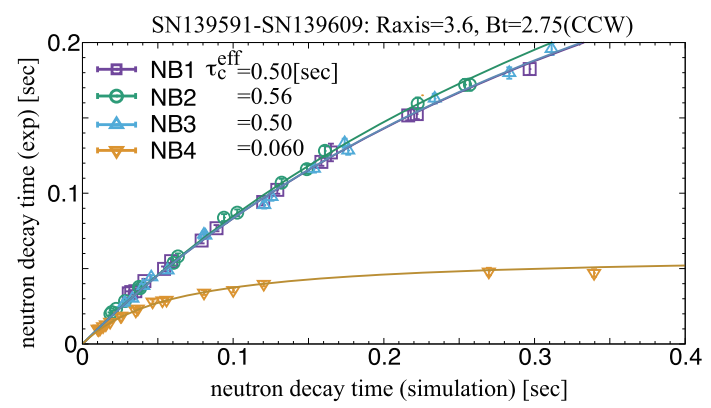

Fig. 5 The measured neutron decay times are plotted against the simulated decay times. The simulated decay time is roughly proportional to $T_{e}^{1.5} n_{e}^{-1}$ as shown in eq. (2).

to the neutron decay time can not be ignored. The auxiliary curves and the confinement time $\tau_{c}$ displayed in Fig. 5 are obtained by the weighted least-square method fitting in $\tau_{n}^{\exp }=\tau_{n}^{\operatorname{sim}} \tau_{c} /\left(\tau_{n}^{\text {sim }}+\tau_{c}\right)$. From Fig. 5 , it is found that the fast ion confinement time of three tangential NBs (NB\#1 - \#3) are longer than that of perpendicular beam (NB\#4). This is because the perpendicular NB fast ions have a poor confinement due to the strong ripple-induced diffusion in LHD [19].

Next, we investigate the relation of the two peak values of the measured and simulated neutron emission rate, $S_{n}^{\text {peak }}(\exp )$ and $S_{n}^{\text {peak }}(\operatorname{sim})$. For simplicity, we assume the plasma parameters and the fast ion birth rate, $r_{f}\left[\mathrm{~m}^{-3} \mathrm{~s}^{-1}\right]$, are constant in time. The kinetic energy of the test fast ion is highest immediately after birth. Therefore, the neutron emission rate, which is made by the test fast ion, is also highest at the time. From eq. (6), the neutron emission rate generated by the test ion, which has the injection energy $E_{0}$, can be expressed as $\int d V n_{D} r_{f} d t\langle\sigma v\rangle_{b t}\left(E_{0}, T_{i}\right)$, where $\int d V$ indicates the volume integration and the velocity distribution is assumed to be the delta function. Since this value decays exponentially with the time constant $\tau_{n}^{\text {exp }}$, the neutron emission rate at $t_{0}$, which is yielded by the test fast ion injected at $t$ becomes $\int d V n_{D} r_{f} d t\langle\sigma v\rangle_{b t}\left(E_{0}, T_{i}\right) \exp \left[\left(t_{0}-t\right) / \tau_{n}^{\exp }\right]$. Therefore, the peak value $S_{n}^{\text {peak }}(\exp )$ can be expressed as

$$
\begin{aligned}
& S_{n}^{\text {peak }}(\exp )=C_{\exp } \int_{0}^{t_{0}} \exp \left(\frac{t_{0}-t}{\tau_{n}^{\text {sim }} \tau_{c} /\left(\tau_{n}^{\text {sim }}+\tau_{c}\right)}\right) d t, \\
& C_{\text {exp }} \equiv \int n_{D} r_{f}\langle\sigma v\rangle_{b t}\left(E_{0}, T_{i}\right) d V,
\end{aligned}
$$

where $t_{0}$ indicate the duration of the short pulse NB injection. In the present case, $t_{0}=0.04 \mathrm{sec}$.

Similarly, simulated peak value $S_{n}^{\text {peak }}(\operatorname{sim})$ can be expressed as:

$$
\begin{aligned}
& S_{n}^{\text {peak }}(\operatorname{sim})=C_{\text {sim }} \int_{0}^{t_{0}} \exp \left(\frac{t_{0}-t}{\tau_{n}^{\text {sim }}}\right) d t, \\
& C_{\text {sim }} \equiv \int n_{e} r_{f}\langle\sigma v\rangle_{b t}\left(E_{0}, T_{i}\right) d V,
\end{aligned}
$$

where the electron density $n_{e}$ appears instead of the

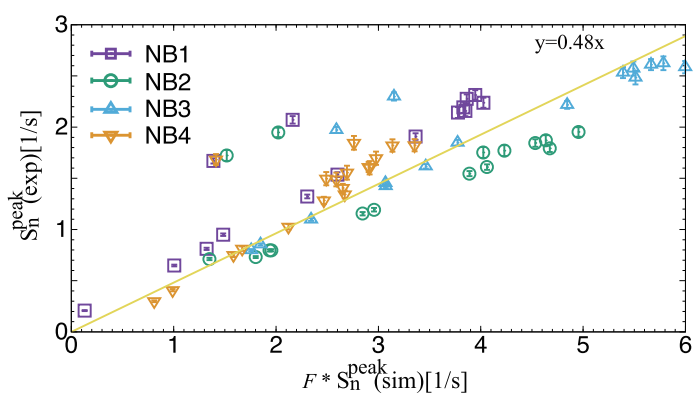

Fig. 6 The measured peak value of the neutron emission rate $S_{n}^{\text {peak }}(\exp )$ is plotted against $\mathcal{F} S_{n}^{\text {peak }}(\operatorname{sim})$.

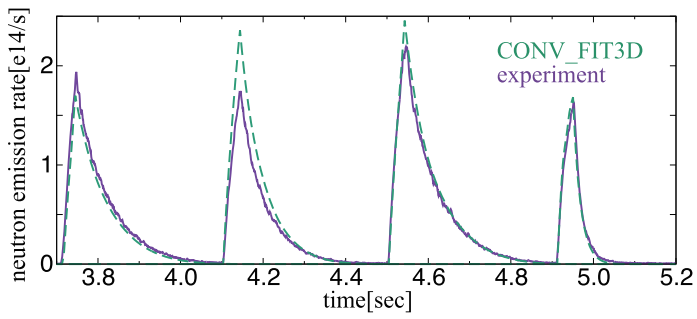

Fig. 7 Evolutions of the measured and simulated neutron emission rates are shown. Simulation assumes $n_{D} / n_{e}=0.5$, $n_{H e} / n_{e}=0.056$, and $n_{C} / n_{e}=0.065$. In addition, three tangential NBs decay with $\tau_{c}=0.5 \mathrm{sec}$ and the perpendicular NB decays with $\tau_{c}=0.06 \mathrm{sec}$.

deuteron density $n_{D}$ because of the assumption of the pure $\mathrm{D}$ plasma. With the assumption that the deuteron density ratio to the electron density is uniform, from eqs. (10) and (13) the ratio of two peak values have the following relation:

$$
\begin{aligned}
& \frac{S_{n}^{\text {peak }}(\exp )}{S_{n}^{\text {peak }}(\operatorname{sim})}=\frac{n_{D}}{n_{e}} \mathcal{F}, \\
& \mathcal{F} \equiv \frac{\tau_{c}}{\tau_{n}^{\text {sim }}+\tau_{c}} \frac{1-\exp \left(-t_{0} /\left[\tau_{n}^{\text {sim }} \tau_{c} /\left(\tau_{n}^{\text {sim }}+\tau_{c}\right)\right]\right)}{1-\exp \left(-t_{0} / \tau_{n}^{\text {sim }}\right)}
\end{aligned}
$$

Figure 6 shows the relation between $S_{n}^{\text {peak }}(\exp )$ and $S_{n}^{\text {peak }}(\operatorname{sim})$. The horizontal axis denotes $\mathcal{F} S_{n}^{\text {peak }}$ (sim) and the vertical axis denotes $S_{n}^{\text {peak }}$ (exp). From eq. (14), the gradient of the fitting line, which is shown in Fig. 6, indicates $n_{D} / n_{e}$. The measured value of $\mathrm{H}-\mathrm{He}$ ratio is approximately $\left(n_{H}+n_{D}\right) /\left(n_{H}+n_{D}+n_{H e}\right) \sim 0.9$. Here, it is assumed that there are only two impurities, ${ }_{2}^{4} \mathrm{He}$ and ${ }_{6}^{12} \mathrm{C}$, there are no protons, and that $n_{D} /\left(n_{D}+n_{H e}\right)=0.9$. From these assumptions and $n_{D} / n_{e}=0.5$, we can estimate $n_{H e} / n_{e} \sim 0.056, n_{C} / n_{e} \sim 0.065$, and $Z_{\mathrm{eff}} \sim 3.1$. Since the typical value of $Z_{\text {eff }}$ in LHD plasmas $\left(n_{e} \sim 10^{19} \mathrm{~m}^{-3}\right)$ measured by the extreme ultraviolet (EUV) spectrometer $[20,21]$ is $Z_{\text {eff }} \sim 2-4$, it can be considered that the estimation of $Z_{\mathrm{eff}}$ is valid.

By using these ion ratios, the neutron emission rate is re-calculated by CONV_FIT3D.

Figure 7 shows the evolutions of the measured and 
simulated neutron emission rates. Solid and dotted curves indicate the experiment and the simulated neutron emission rate. Simulation assumes $n_{D} / n_{e}=0.5, n_{H e} / n_{e}=$ 0.056 , and $n_{C} / n_{e}=0.065\left(Z_{\mathrm{eff}} \sim 3.1\right)$. Additionally, fast ions from three tangential NBs decay with time constant $\tau_{c}=0.5 \mathrm{sec}$ and the perpendicular NB decays with $\tau_{c}=$ $0.06 \mathrm{sec}$. With these assumptions, we can obtain a consistent result with the experiment result. It is noted that since the ion ratios are not always constant in this series of experiments, an accurate prediction can not be obtained by this estimation.

\section{Conclusion}

In this paper, a series of experiments and simulation analyses have been performed to investigate the confinement time of NB fast ions in LHD. We observed the neutron emission rate itself and the decay time of the neutron emission rate, which is generated by DD fusion reaction, in the short-pulse NB injection experiments. In addition, to analyze the LHD experiment data, CONV_FIT3D code, which is a NB heating component of TASK3D-a, has been extended. In LHD, since the beam-thermal fusion reaction is dominant, CONV_FIT3D code implements the beamthermal fusion cross-section derived by Mikkelsen [15]. The validity of the simulation result of the neutron estimation has been checked through a benchmark test with FBURN [17] code and TASK/FP [8].

The decay of the neutron emission rate after the NB is turned off comes from two physics factors. One is the reduction of the DD fusion cross-section due to the slowing down of the NB fast ions. The other is the reduction of the fast deuteron density due to the fast deuteron transport. CONV_FIT3D, which is based on the classical Coulomb collision theory, can only describe the contribution of the fast ion slowing down. Therefore, the differences between measured and simulated neutron decay time indicate the fast ion confinement time as shown in eq. (1).

Figure 5 shows the relation between the measured and the simulated neutron decay times. Owing to the fast deuteron transport, the simulated neutron decay times separate from the measured values as the fast deuteron slowing down time increases. From these data, the fast deuteron confinement time in each beam can be roughly estimated as shown in Fig. 5. Additionally, from the measured and simulated peak values of the neutron emission rate, ion density ratio can be roughly estimated as shown in eq. (14). Finally, by using the estimated fast ion confinement time and ion density ratio, we can obtain a simulation result of the neutron emission rate similar to the measured emission rate as shown in Fig. 7. It is noted that this estimation includes the assumption that the deuteron density ratio is the same in each shot.

In this paper, the results for single magnetic configuration are shown. Through analyses with several magnetic configurations, the magnetic configuration dependence of the fast ion confinement time will be investigated in future works.

\section{Acknowledgments}

This work is supported partly by LHD project budgets (NIFS07KLPH004 and NIFS14KNTT025). This work has been performed on "Plasma Simulator" (FUJITSU FX100) of NIFS with the support and under the auspices of the NIFS Collaboration Research program (NIFS16KNSR005).

[1] Y. Takeiri, IEEE Trans. Plasma Sci. 46, 2348 (2018).

[2] M. Osakabe, M. Isobe, M. Tanaka et al., IEEE Trans. Plasma Sci. 46, 2324 (2018).

[3] Y. Takeiri, IEEE Trans. Plasma Sci. 46, 1141 (2018).

[4] M. Osakabe, Y. Takeiri, T. Morisaki et al., Fusion Sci. Technol. 72, 199 (2017).

[5] M. Isobe, K. Ogawa, T. Nishitani et al., IEEE Trans. Plasma Sci. 46, 2050 (2018).

[6] J. Strachan, P. Colestock, S. Davis et al., Nucl. Fusion 21, 67 (1981).

[7] W. Heidbrink, J. Kim and R. Groebner, Nucl. Fusion 28, 1897 (1988).

[8] H. Nuga, R. Seki, S. Kamio et al., Nucl. Fusion 59, 016007 (2019).

[9] M. Isobe, K. Ogawa, H. Miyake et al., Rev. Sci. Instrum. 85, 11E114 (2014).

[10] S. Murakami, N. Nakajima and M. Okamoto, Trans. Fusion Technol. 27, 256 (1995).

[11] M. Sato, S. Murakami, A. Fukuyama et al., Proc. 18th Int. Toki Conf, 2008.

[12] P. Vincenzi, T. Bolzonella, S. Murakami et al., Plasma Phys. Control. Fusion 58, 125008 (2016).

[13] M. Yokoyama, R. Seki, C. Suzuki et al., Nucl. Fusion 57, 126016 (2017).

[14] T.H. Stix, Plasma Phys. 14, 367 (1972).

[15] D. Mikkelsen, Nucl. Fusion 29, 1113 (1989).

[16] H.-S. Bosch and G. Hale, Nucl. Fusion 32, 611 (1992).

[17] K. Ogawa, M. Isobe, T. Nishitani et al., Plasma Phys. Control. Fusion 60, 095010 (2018).

[18] B. Braams and C. Karney, Phys. Fluid B 1, 1355 (1989).

[19] S. Murakami, H. Yamada, M. Sasao et al., Fusion Sci. Technol. 46, 241 (2004).

[20] H. Zhou, S. Morita, M. Goto et al., Rev. Sci. Instrum. 81, 10D706 (2010).

[21] X. Huang, S. Morita, T. Oishi et al., Plasma Fusion Res. 10, 3402036 (2015). 